\title{
Agua si, agua tampoco
}

Oscar Pastor

opastorp@gmail.com

\section{Resumen}

Para las autoridades y los representantes de la Sociedad Civil de la ciudad de Arequipa, la firma del convenio con la SMCV ${ }^{1}$ a mediados del año 2006, fue un hecho histórico, unos años más y se producirá el $100 \%$ de agua potable y se tratará el $100 \%$ de las aguas residuales de toda la ciudad. El documento suscrito con el aval del Premier de la República, no hizo mención a la ampliación de cobertura, llevar el agua potable y los servicios de saneamiento hasta el domicilio.

La prensa en general y la escrita en particular actúo como correa de trasmisión entre las diferentes ideas, intereses y posiciones de los grupos que se van definiendo de acuerdo al desarrollo de los hechos. La historia reciente, construida públicamente nos muestra esta particularidad.

Para analizar un hecho sin interés manifiesto, donde el investigador es simultáneamente informante, se requiere dejar de lado la subjetividad y asumir el convencimiento que la investigación no tiene como propósito presentar juicios de valor, son otros los que deben construirlos. Es natural que cada actor defienda sus ideas y es natural también que tenga estos argumentos presentes cuando evalúe su participación. Recurrir a los periódicos y extraer de allí los hechos es una forma de evitar la subjetividad.

\section{Arequipa}

Un gran volcán de forma cónica, flanqueado por otros dos de caprichosas figuras es lo primero que impacta al visitante, repuesto del shock, el inmenso cielo azul lo vuelve a sacudir, el color blanco de sus construcciones de sillar y la campiña dentro de la ciudad, revolotearán en su mente por un buen tiempo.

Así es Arequipa de sorprendente, como sus paisajes sociales.

El protagonismo sureño y el recuerdo de notables personajes de reconocida trayectoria, les ha dado a los arequipeños una particular forma de sentir la tierra, "no en vano se nace a las faldas de un volcán", se puede leer en uno de los portales de ignimbrita, que embellecen la tradicional plaza del distrito de Yanahuara y proyecta una tranquilidad que Arequipa esta perdiendo.

La ciudad tiene una población cercana al millón de habitantes, está asentada sobre $200 \mathrm{~km}^{2}$ de tierra volcánica y fértil, irrigada por el río Chili que provee más del $90 \%$ del agua para consumo humano. El río llega a la ciudad desde las alturas andinas -en realidad desde la represa de Aguada Blanca-, en medio de un cañón profundo que separa a los volcanes Misti y Chachani, cruza la ciudad, para efectos prácticos, de este a oeste algo más de $11 \mathrm{~km}$ hasta el distrito Uchumayo, donde gira bruscamente al norte y se encañona nuevamente, para retornar a su dirección natural y desembocar como todos los ríos de la costa peruana, al océano pacífico. La topografía se encarga de darle a la ciudad una pendiente moderada. El Google Earth es de gran ayuda para estos casos.

${ }^{1}$ Ver Anexo I: Lista de Acrónimos y personajes

1) Page Vol1, No 2/2013 ABCResearch Alert 


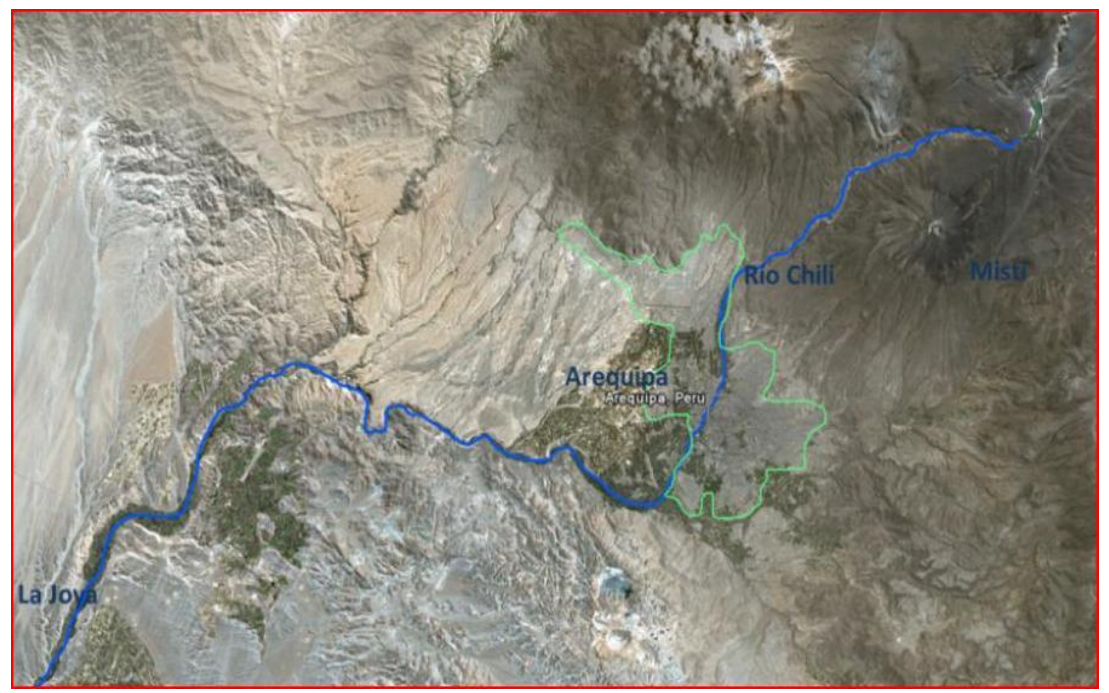

El río Chili divide a Arequipa en dos zonas: norte y sur; con respecto a las montañas tutelares la división corresponde a las zonas alta y baja. Las ubicadas al pie de los volcanes tienen una topografía ondulante y algunas veces muy accidentada. Las aguas de lluvia y las domésticas fluyen hacia el río con naturalidad, la vida en sus aguas hace un buen tiempo es mínima.

Dicen, no con apego a la verdad, que el Cono Norte ${ }^{2}$ de la ciudad es la zona más populosa de Arequipa; tal vez por que hace años viene siendo poblada con gran velocidad por arequipeños y migrantes de la sierra sur del país. Hacia el oeste del Cono Norte y alejada en altura y distancia de las riberas del rio Chili, está la zona más deshabitada de Arequipa, que colinda con otra de importancia agrícola. El GRA está construyendo por allí una moderna carretera que unirá Arequipa con el desierto de La Joya, sector irrigado por el río Chili, cuando orienta su curso nuevamente al oeste.

Las extensas tierras rojizas y eriazas, ondulantes y lejanas, expuestas al sol y a los vientos en la zona despoblada de la ciudad, son atractivas para quienes no pierden la esperanza de convertir los terrenos eriazos en productivos, para ello necesitan agua y es seguro que el río Chili no las abastecerá.

Sólo un desinformado diría que Arequipa está en un desierto, si es que no ha observado que toda la costa peruana y el norte de Chile son desérticos; sólo un informado diría que el río Chili no existe, porqué conoce como pocos, que el río le debe sus aguas a una cuenca regulada por un sistema de represas, que depende de las lluvias para garantizar el bienestar humano y económico de Arequipa.

A pesar de las dificultades naturales, la ciudad tiene una cobertura de agua potable del $90 \%{ }^{3}$, de los más de 40 millones de $\mathrm{m}^{3}$ de agua potable que SEDAPAR produce al año, alrededor de 25 millones de $\mathrm{m}^{3}$ de aguas residuales se vierten a sus quebradas y especialmente al río Chili, la vista y el olfato son suficientes para comprobar el estado de contaminación del río, que actúa contra su voluntad como un gran colector de desagües; sin un sistema de evacuación de aguas residuales la vida en la ciudad sería imposible, lo que es probable que suceda si no se detiene la contaminación. Muy poco se puede decir de la calidad del agua tratada en la PTAR de Chilpina, ubicada hacia el sur oeste de la ciudad, sus efluentes son utilizados en la agricultura y ganadería, un buen paseo por allí le bastaría para confirmarlo.

2

Se ubica a 10 km o más de la margen derecha del rio, cercana al volcán Chachani, el aeropuerto internacional Rodríguez Ballón y la carretera que une Arequipa y Puno, es su principal referencia

3 de cada 10 personas tiene agua potable en su domicilio, en la ciudad de Arequipa el promedio de continuidad de servicio de agua potable es 22 de horas al día 


\section{Los medios de comunicación}

No es una exageración decir que en la ciudad se producen todos los días alrededor de 300 horas de radio, 30 horas de televisión regional, 30 paginas de periódicos locales y otras tantas de alcance nacional dedicados a las noticias, la noble profesión del periodismo requiere más de 1,000 horas-hombre-día para buscar, descubrir, fabricar, mantener y presentar la realidad, o más bien su proceso de construcción, que de manera intencional o no, orienta, confunde, defiende, ataca, muestra, esconde y pocas veces reconoce los éxitos. Que sería de nosotros sin ellos y de ellos sin los generadores de noticias, y de los newsholic o consumidores de noticias. Con significativas diferencias esta ecuación se repite en las capitales de provincia y distritos, los que tienen una alta vinculación noticiosa con la capital de la región, los medios de la ciudad de Arequipa invaden la cotidianeidad todos los días del año.

Los medios convierten hechos en intereses, sean estos de valor social, económico y político entre otros; por tanto, requieren de actores sociales, económicos y políticos como generadores de hechos, aunque parezca una verdad de perogrullo, los hechos políticos los generan los políticos, que necesitan visibilidad y exposición para que su comportamiento transite entre los hechos y sus intereses, a mayor representatividad política mayor necesidad de visibilidad e influencia. Medios y política se atraen, este coctel social se abona con los intereses económicos, pocas veces expuesto con transparencia, entender este triangulo requiere una lectura crítica, no solo basada en el recuerdo, sino en evidencias que se mantienen inalterables en el tiempo y que la custodian precisamente los medios escritos. Los periódicos arequipeños son la fuente que sustenta esta investigación.

Analizar un hecho o un interés especial, del cual uno ha sido en algún momento actor principal tiene sus ventajas, en tanto se ha tenido acceso a información que no siempre es de conocimiento público, convertirse en su propio informante confronta el análisis con la subjetividad, la tentación de suprimir o modificar hechos es una opción irrenunciable, más aún cuando de por medio hay un interés; basta recordar que en las cultura ágrafas se cortaba la lengua a quienes guardaban la historia, en los tiempos de la escritura se quemaron libros y se prohibió el acceso a determinada información, precisamente para modificar los hechos, por interés. Una estrategia para diluir esta posibilidad es centrarse en aquello que está publicado en los medios escritos de acceso público, fuente de construcción de la realidad, que aceptada por la sociedad se convierte en un stock que alimenta los siguientes procesos.

\section{Cuestiones previas: la Planta de Tratamiento de Agua Potable}

A mediados del gobierno del presidente Alan García, el Perú asistía con asombro al cambio acelerado de la economía, los altos precios de los minerales dejaron de ser información especializada, tampoco lo serían las utilidades generadas por la actividad minera, las voces que reclamaban mayor participación del gobierno eran cada vez más frecuentes y su tono cada vez más confrontacional. La sociedad y las empresas mineras observaban con impaciencia el desarrollo de los acontecimientos, a la par las autoridades políticas y dirigentes sociales de Arequipa negociaban, con participación de representantes del más alto nivel del gobierno central, un Convenio con la SMCV con un solo punto en agenda: Resolver el problema de agua potable y aguas residuales de la ciudad. La SMCV se comprometió a financiar los estudios de las futuras plantas de tratamiento de agua potable y de aguas residuales.

3|Page Vol 1, No 2/2013 ABCResearch Alert 
Este breve relato ha cumplido con el rigor histórico de apostillar a los que suscribieron el acta, en este caso en particular el proceso de negociación tuvo un invitado ausente: SEDAPAR. Este olvido o deseo de no compartir el control de los hechos, quizá explique por qué se optó por encargar a la SMCV la construcción de la PTAP, tan solo comparar los indicadores de cobertura y continuidad contra los de tratamiento de aguas residuales y oler el río Chili hubiesen sugerido otra decisión, pero así estuvieron las cosas y después de dos años de completo silencio, se empezó a insistir desde SEDAPAR el cumplimiento del compromiso. El Anexo II presenta una cronología de los olvidos.

La SMCV convocó a un concurso internacional para elaborar el Expediente Técnico de ambas plantas y se olvidó de participar a la contraparte que suscribió el documento (ver Anexo II), mucho menos a SEDAPAR, quién tal vez la habría obligado a cumplir el ciclo de proyectos de inversión pública ${ }^{4}$ y convocar los estudios que corresponden a la etapa de pre inversión, donde se estudian las opciones técnicas, económicas, de ubicación, etc. En este nivel se escoge la propuesta más recomendable y luego se desarrollan los estudios con mayor profundidad, como el Expediente Técnico; sin embargo, aquí se empezó por el final.

Lo cierto es que los estudios de la PTAP fueron realizados con total hermetismo por la SMCV, luego se produjo un acompañamiento cercano y efectivo de SEDAPAR hasta que se empezaron las obras, y el hermetismo volvió para quedarse. Pasado el tiempo y corregidos o profundizados algunos errores, a mediados del año 2012, el presidente Ollanta Humala en una breve ceremonia inauguró la PTAP llamada desde el Expediente Técnico "La Tomilla II", luego de varios meses de la visita presidencial, SEDAPAR aún no recauda ingresos por la venta de agua potable a las nuevas conexiones que con expectativa esperaba que la nueva PTAP empezase a producir.

A ciencia cierta, nadie dijo cuánto costará producir agua potable en la nueva PTAP, tampoco si se han solicitado nuevas tarifas para afrontar los costos de operación. Varios años después de iniciado todo este largo proceso, el 05 de diciembre del $2012^{5}$ un periódico de circulación regional recordando el olvido, lanzo el siguiente titular: "Sedapar paga S/. 23 millones por operar planta de agua № 2". Para ser justo diré que las buenas noticias también se olvidan, el valor de la "Tomilla II" es similar o mayor a todo el valor de los activos contabilizados de SEDAPAR.

Hay olvidos intencionales y circunstanciales, un claro ejemplo es que varias autoridades que suscribieron el convenio, dejaron de serlo al concluir el periodo para el cual fueron elegidos, el vacío fue llenado inmediatamente por nuevos actores, que inmediatamente empezaron a informar con interés los avances de los hechos.

No es difícil desviarse del objeto de la investigación cuando se encuentran elementos de igual o mayor importancia, nos pasa siempre. Sin el propósito de justificar esta navegación, diré que los hechos y los intereses que rodean la inversión de la PTAP son un antecedente indesligable para entender la otra planta, la PTAR, esa que nadie quiere tener cerca a su casa, que genera rechazo, por algo tratan aguas 4

4 http://www.bibliotecavirtual.uni.edu.pe/pdf/gestion\%20publica/SNIP\%20PARA\%20TODOS.pdf. Pag 42

5 http://www.larepublica.pe/05-12-2012/sedapar-paga-s-23-mlls-por-operar-planta-de-agua-n-2

4|Page Vol 1, No 2/2013 ABCResearch Alert 
residuales, nadie quiere convivir con los residuos, menos con los residuos humanos, apestan, traen enfermedades, degradan el status habitacional.

El progreso exige tener agua potable y desagüe en casa, todavía no incluye el tratamiento de esas aguas, si no trae beneficios visibles, directos, nadie tiene por que apreciar algo que no le genera valor, más aún cuando desde lo más alto del gobierno se emiten mensajes que no ayudan a entender la complejidad del problema, como el que popularizó el gobierno del presidente Alan García, "Sin agua no hay democracia", que sustentó el "Programa de Inversiones Agua para Todos", y llevó al gobierno a enfatizar las inversiones para disminuir la brecha de cobertura. Tal vez Saneamiento para todos, Tratamiento de aguas residuales para todos, habrían direccionado los recursos de manera diferente.

\section{Historia de nunca acabar}

Regresemos a la otra planta, la de aguas residuales, la PTAR.

Por esas cosas que tiene el destino, los responsables de los estudios se olvidaron de consultar con la población la ubicación de la PTAR. El primer lugar escogido fue Tunales, cerca al punto de mayor descarga de aguas residuales sobre el río Chili, a unos cientos de metros donde gira bruscamente hacia el norte. Un grupo de alcaldes con un reconocido nivel de representatividad se opuso a la propuesta de ubicación presentada por la SMCV, la justificación fue simple; no se puede cambiar el uso de los terrenos agrícolas. SEDAPAR aceptó sin mayores problemas y luego inició la busca de una nueva ubicación, según se dijo la SMCV ya había cumplido con presentar los estudios.

Un titular del 02 de julio del 2009 da comienzo a una gran historia: "Planta de aguas residuales tendrá nueva ubicación", el anuncio lo hace el AP de Arequipa, Simón Balbuena, que "prefirió mantener el sitio exacto en reserva, los funcionarios de SEDAPAR serán los responsables de presentar los resultados el próximo 20 de julio", continua "la construcción permitirá un ahorro de 60 millones de soles con respecto a la primera propuesta". ¿Cómo llegó la información a dicha autoridad?, ¿por qué la noticia no la dio un representante de SEDAPAR?, ¿en qué momento se aceptó que la SMCV se desligue del estudio?, son algunas de las interrogantes cuyas respuestas son muy amigas del silencio.

No pasaron más de veinte días después de la promesa, para que el Gobernador Regional Oscar Achata ofrezca su ayuda con el gobierno central, del cual es su representante y partidario. Por ahora las noticias parecen buenas, ese mismo día Juan Manuel Guillén, Presidente del GRA anuncia "la posibilidad de construir Plantas descentralizadas en diferentes distritos de la ciudad". Todo parece bajo control, el 24 de julio la consultora contratada por SEDAPAR expone el proyecto formalmente en el GR. De las cinco alternativas consideradas, que incluye el proyecto Pampa Estrella ${ }^{6}$, se dijo que "Ias que tienen mejor disponibilidad y no cuentan con limitaciones legales son las 25 has. del terreno Los Hurtados, ubicado detrás de la Curva del Cura en el distrito de Uchumayo y Pampa Escalerilla ubicada en el distrito de Cerro

6

6 El 04 de enero del 2002 un diario de la ciudad público: "Proyecto La Estrella es la solución al problema", se dijo también que "En 1999 SEDAPAR suscribió el contrato de préstamo con la KFW de Alemania para la ejecución de dicho proyecto; sin embargo, después de casi 4 años debido a la insolvencia económica de SEDAPAR que no le permite aportar la contrapartida requerida, el crédito alemán de 42 millones de dólares aun no puede hacerse efectivo. El año 2000 la firma Fitchner culminó la elaboración del expediente técnico y un año después los terrenos de Pampa Estrella quedaron saneados a favor de SEDAPAR".

5|Page Vol1, No 2/2013 ABC Research Alert 
Colorado". El AP de Arequipa, el presidente del GRA y el gobernador al alimón anunciando hechos presentes y futuros, ¿¿cuáles serán sus intereses?

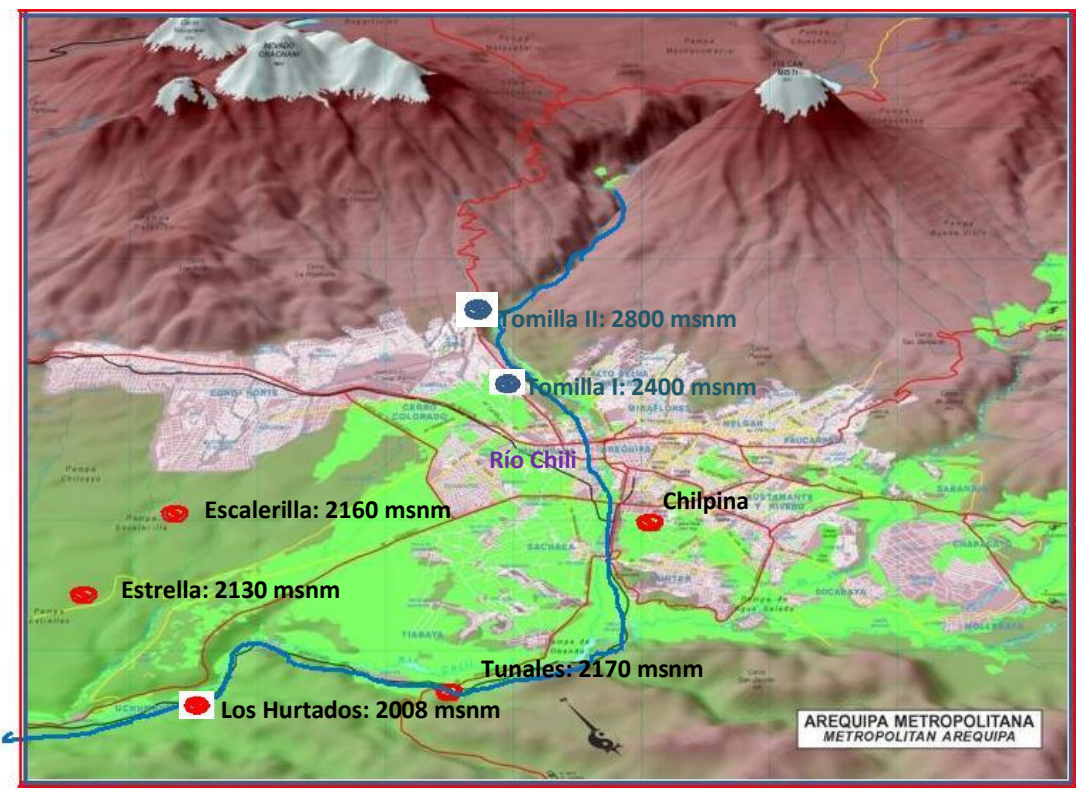

La discusión y desacuerdos de los actores involucrados en el proyecto de las PTAR, tiene su origen en la ubicación y el poder que desarrolla cada uno de ellos alrededor de sus intereses. Las herramientas visuales disponibles en la web ayudan de manera significativa a tener una visión panorámica de las propuestas de ubicación de las PTAR y de la nueva PTAP.

Las buenas noticias vienen en letras pequeñas, las otras también llegaron, el domingo 26 de julio, "los pobladores de Uchumayo enterados de la ubicación, en una reunión de emergencia arrinconaron al alcalde y sus regidores y acordaron rechazar el gran proyecto de desagüe, dejando sin piso a SEDAPAR. Dura tarea le espera al gobierno regional", para mayor veracidad de la noticia, aparece, como se repetirá en varias ocasiones más, un pronunciamiento oficial de la MD de Uchumayo, reconociendo la necesidad de la PTAR, invocando que retornen al proyecto Pampa Estrella.

Sólo se necesitaron cuatro días para tener los equipos, los capitanes, algunos jugadores presentes y ausentes, de un partido que aún no termina y una historia que ha sido contada con rigurosidad cronológica por los medios, donde valen por igual la versión oficial, las posiciones interesadas, los comentarios, el doble discurso, el discurso ausente, el que se reitera y el que se olvida, el que se comenta con legítimo interés, con desconocimiento, maldad y hasta con discursos y entrevistas que coquetean con el delito de Falsedad Genérica ${ }^{7}$

Como todo conflicto, este ha tenido su etapa embrionaria, de desarrollo, explosión, madurez y actual indiferencia, de todo ello dan cuenta los medios y para su relectura es conveniente tomar nota que toda palabra, toda expresión, todo gesto, toda participación pública, es portadora de una carga ideológica que oculta intereses de diversa índole.

En la ciudad de Arequipa se concentran todos los poderes del estado nacional, regional y local, es capital de la provincia y sede del gobierno regional, la provincia tiene 29 distritos, 13 de ellos se ubican en lo

7

7 Decreto Legislativo № 635, Código Penal: Art 438

6|Page Vol1, No 2/2013 AB R Research Alert 
que se denomina Arequipa Metropolitana y son atendidos por SEDAPAR, los accionistas de la empresa suman 33 entre alcaldes provinciales y distritales, en el directorio de la empresa participan dos representantes municipales, uno de ellos preside el directorio, un representante del GRA y otros dos de la CC y el CIP - Consejo Departamental de Arequipa.

A finales del mes de julio del 2009, Oscar Pastor, GG de SEDAPAR informa los avances de los estudios y la evaluación técnica de las opciones. El AD de Uchumayo, Hernán Alegre rechaza cualquier posibilidad de construir la PTAR en el terreno Los Hurtados, el presidente del GR fija como fecha límite el 03 de agosto para que SEDAPAR le presente el cronograma definitivo del proyecto, noticia que es acogida en varios medios, se publica un nuevo pronunciamiento oficial de la MD de Uchumayo, que ratifica su rechazo a Los Hurtados y su aceptación a la ubicación en Pampa Estrella que también está en su distrito.

No había pasado más de treinta días desde el primer anuncio de retomo del proyecto, para que aparezca un nuevo actor, con un pronunciamiento en formato oficial, el Comando de Lucha del Cono Norte, integrado por tres instituciones algunas veces confrontadas, pide "no ceder ante los enemigos del desarrollo, de gente que está al margen de la ciencia y la tecnología, escudándose detrás del pueblo, es urgente construir las dos plantas". A más de uno le llamará la atención, el espacio que han logrado los representantes de los pueblos ${ }^{8}$ agrupados en diferentes organizaciones que pelean por el control político del Cono Norte, sus demandas por servicios públicos les da a sus dirigentes visibilidad en los medios, anuncian de manera recurrente su capacidad de movilización de hasta 200,000 personas sustentando así su importancia en términos políticos y sociales.

Los personajes y sus discursos en esta historia empiezan a mostrarse. Los políticos con una posición cambiante, los representantes sociales y las autoridades de la cuenca baja con sus interés particulares, los técnicos encapsulados en su esquema informativo, un opositor que personaliza la protesta, tres grandes ausentes, uno local, los otros dos que supieron mantenerse al margen, con mandato nacional y los medios de comunicación como correas de trasmisión informativa. A esta figura incompleta, formada por grupos políticos, sociales, técnicos y mediáticos, se unirán otros actores personales e institucionales que se hacen visibles en los medios, que usan la contradicción y confrontación para asegurar su participación y ampliar su cobertura en los medios.

La declaración del AD de Cerro Colorado, Manuel Vera, que cuestiona las opiniones que no respetan criterios técnicos, precedió al editorial de un tradicional periódico arequipeño que fija su posición de respaldo a la opinión técnica, luego vendrían otros. Esto exacerba la respuesta del AD de Uchumayo que declara "el pueblo ha decidido rechazar la propuesta y la respaldo" y la de Mario Jacobo, presidente del FREDECIR, que le da un plazo perentorio al alcalde opositor para que cambie de opinión, sino "harán plantón y lo pasearán en burro".

Mientras estas fricciones tienen lugar en los medios, el director de SEDAPAR, Hugo Rosand anuncia la presentación del cronograma. El GG del GRA, Berly Gonzales declara que se destinarán 75 millones de soles para la construcción de las Plantas, y que "optarán por Escalerilla, en caso que los alcaldes y SEDAPAR no fijen el lugar hasta el 6 de agosto". Este inofensivo anuncio del GG, que carece de

${ }^{8}$ Los dirigentes llaman así a los asentamientos con diferentes niveles de construcción, agrupados por razones geográficas, históricas y políticas.

7|Page Vol1, No 2/2013 ABCResearch Alert 
capacidad para decidir unilateralmente asuntos de esta importancia, juntará solapadamente por largo tiempo a actores políticos regionales y locales especialmente del distrito de Cerro Colorado, con los representantes sociales del Cono Norte.

El AP de Arequipa garantiza sus aportes económicos. EI PD de SEDAPAR, James Fernández refiriéndose a la PTAR Chilpinilla asegura que "Ias nuevas tendencias no permiten construir una planta asi", y agrega que "si se cumple con los requisitos, a fines de octubre se estaría licitando la obra".

Los plazos en asuntos técnicos y sociales son un buen ingrediente noticioso, es la excusa perfecta para asegurar una nueva entrega de hechos, si la promesa se cumple, la nota periodística está asegurada, también lo está si no se cumple; en este último caso, la noticia llega en forma de crítica. SEDAPAR el 08 de agosto solicitó cuatro días más para presentar la propuesta, esta demora excesiva es usada de excusa por el Presidente del GR para "solicitar a SEDAPAR la viabilidad y ejecutar la PTAR de Escalerilla, hasta que se decida la ubicación de la otra planta".

La historia toma otro giro con la participación de especialistas locales en saneamiento y diseño de $\mathrm{PTAR}^{9}$, el proyecto de Pampa Estrella utilizará "tecnología natural, que generará biogás, abonos, energía hidroeléctrica, y su vida útil será mayor", dijeron a medio local. EI AD de Uchumayo pide consulta popular, herramienta perfecta de dilación de las decisiones, el pueblo quiere participar y no se le puede negar ese derecho.

Como respuesta, el AD de Cerro Colorado hace de conocimiento público que "si no aprueban el proyecto, o si se demoran más, nosotros vamos a insistir en la construcción de la Planta Escalerilla necesaria para el distrito y para otros aledaños". En esta misma línea el Presidente y funcionarios del GRA y los dirigentes del Cono Norte anunciarán en repetidas oportunidades que tienen presupuesto suficiente para cofinanciar la PTAR, terrenos saneados y licencia social; las declaraciones difieren en montos y plazos, la urgencia justifica cualquier desinformación. En algún momento de pleno convencimiento se dijo que una vez concluida la PTAR de Escalerilla, el distrito de Cerro Colorado se cambiará de nombre a Cerro Verde, en alusión al futuro uso de los efluentes para arborizar el distrito.

La oportunidad de hacerse visible no toca la puerta dos veces, La Joya es un distrito cuya economía depende del río Chili, su AD David Inofuente mediante un pronunciamiento oficial manifiesta que su pueblo no está de acuerdo que se construya la PTAP en Arequipa, si antes no se ha concluido la PTAR, similar opinión tendrán otros representantes de la denominada cuenca baja del Chili, posición que los llevará a exigir la descontaminación del río y defender una ubicación que garantice que el agua será devuelta al cauce, mostrarán su descuerdo con la gestión de SEDAPAR, se acercarán y alejaran a las autoridades según se avizore algún tipo de solución, movilizarán a la población, buscaran que sus opiniones sean escuchadas, cuando les llegue el convencimiento de su notoriedad amenazarán con tomar la carretera panamericana sur y en unos días más tendrán una participación digna de mejores causas. Este nuevo actor está a favor pero en contra de propuestas y soluciones diferentes. 9

Declaran que actúan con el aval del CIP, que tiene un representante en el directorio de SEDAPAR

8|Page Vol 1, No 2/2013 ABC Research Alert 
Unos días después del pronunciamiento de la MD de La Joya, el PD de SEDAPAR manifiesta que "el inicio de las obras debe ser paralelo, sino se contaminará más el río Chili", pide dialogo con el AD de Uchumayo y no utilizar el proyecto como caballito de batalla en las siguientes elecciones. Sobre la ubicación dice que "las críticas no tienen sustento técnico, el único que toma la decisión es SEDAPAR y lo hace para bien o para mal".

Las prácticas discursivas definieron a dos grupos con altos niveles de confrontación, los que aseguran que las decisiones deben ser tomadas por los técnicos y el grupo que reclaman participación social y consulta popular para la ejecución de inversiones. Hay un tercero indeciso, que por esa característica puede estar en ambos grupos simultáneamente y seguir avanzando con su propósito.

Los medios darán cuenta en los siguientes meses de los esfuerzos para iniciar el diálogo con la participación de la Defensoría del Pueblo. El financiamiento será tratado por todas las autoridades sin excepción, unas para publicitar su cumplimiento parcial, otras para exigir que otras hagan lo mismo, para anunciar que por falta de presupuesto no participarán, o para pedir que el gobierno central asuma todo el compromiso, se formaran comisiones, se pedirá explicaciones o se utilizará la falta de compromiso como argumento para justificar demoras o indecisiones en el camino. El dinero es la mejor excusa para hacer o dejar de hacer, para prometer, crear expectativas, unir, atacar, fortalecer o debilitar posiciones. A un pueblo ilusionado le resulta más conveniente estar del lado del que ofrece gastar y se acercará más, si temerariamente la oferta viene acompañada de plazos.

En los últimos seis meses del año 2009, más de 300 publicaciones periodísticas relacionadas a la PTAR inician sus preguntas con los interrogativos Cuándo y Cuánto, el primero sirve para juzgar la rapidez de respuesta, el segundo evalúa la capacidad y la importancia de la autoridad, estas dos palabras marcan posición en la cadena de poder político según se pida o se ofrezca respuestas. La paciencia no es la principal virtud que cultiva el pueblo necesitado y más de uno sabe cómo explotar esta desesperación.

Con la presencia de Guillermo León, Vice Ministro de Saneamiento se renueva la discusión técnica y el uso de categorías como: caudal promedio, carga orgánica, DBO, reactor anaeróbico, filtro biológico, vida útil, TIR o VAN, que ocuparán grandes espacios en todos los medios escritos. La discusión de la relación entre la temperatura y la eficiencia de los procesos en los reactores anaeróbicos de flujo ascendente y la concentración de la carga orgánica a la salida de la futura planta y el valor que tendrá luego de algunos metros de entregada al río que se encargará de completar el proceso, muestran lo improductivo de estas discusiones para la opinión pública. Una forma de convencer es confundir, o de auto convencerse, mientras algunos se aferraran a los criterios técnicos otros los utilizan para invalidar el proyecto.

Algunos días después que se aceptó desechar el sitio denominado Tunales, SEDAPAR contrató a la empresa H\&C para seleccionar la ubicación de la PTAR. EI CIP, los dirigentes del Cono Norte, autoridades políticas declaraban a la prensa y emitían comunicados pidiendo que se seleccione Pampa Estrella. El 21 de septiembre el Presidente del GRA y Miguel Ocharán Gerente Regional de Planificación del GRA declararon que "a pesar de no tener competencia en la elección de la ubicación de la PTAR, apoyan la propuesta del CIP". Tres días después Hugo Campusano, Gerente General de H\&C, responsable de los

9|Page Vol1, No 2/2013 ABCResearch Alert 
estudios manifiesta que "todas las propuestas resuelven el problema, pero por razones técnicas y económicas Los Hurtados y Escalerilla, requieren menor inversión y costos de operación".

Durante la JGA se presentó el informe oficial el 30 de septiembre; el GG de SEDAPAR "precisó las diferencias técnicas y económicas", dijo también que "la obra no podrá ser iniciada mientras no se tenga asegurado su financiamiento". EI PD de SEDAPAR declaró que "no quiso adelantar la alternativa seleccionada para evitar actos de violencia", al finalizar la reunión el AD de Miraflores, Luis Aguirre dijo que "Ias autoridades deben respetar la decisión técnica y no dejarse llevar por el momento", Alfredo Zegarra, AD de José Luis Bustamante y Rivero pidió "no dejarse presionar por gente que actúa sin argumentos", el AD de Uchumayo que participó de la reunión mantuvo su postura.

Ese mismo día en las afueras del local de SEDAPAR "los pobladores del Cono Norte, exigieron en la calle con banderolas, la construcción de la PTAR", y en Uchumayo los pobladores bloquearon la carretera panamericana sur "impidiendo el paso de 300 vehículos que formaron más de $3 \mathrm{~km}$ de cola en el peaje". Este clima de convulsión trató de ser amainado con un nuevo comunicado a la opinión pública, SEDAPAR reconocía la libertad del consultor para evaluar las propuestas observadas por la OPI y que cumplirá con el cronograma que presentó a las autoridades.

José Pinto decano del CIP dijo una semana antes de la JGA, que "SEDAPAR sólo crea zozobra, Pampa Estrella es idónea, Hurtados carece de licencia social como Tunales", igual discurso tuvo el Decano del

CAA, Ciro Rojas, que dijo que "la Planta no puede hacerse en bases a caprichos, el área es una reserva paisajista en el ingreso a Arequipa, Pampa Estrella no requiere modificar nada",

La discusión ahora se centrará en saber si el terreno es reserva paisajista o no, el 12 de septiembre SEDAPAR entrega los estudios a la MD de Uchumayo, el mismo día el CAA dice que "los Hurtados se ubica en una zona paisajística según el Plan Director de Arequipa"; Clark Velando del CIP pide conservar el terreno de uso agrícola. "Perro del Hortelano" titula un editorial. El 02 de octubre, el AP de Arequipa declara que los terrenos no son agrícolas. La zonificación de Los Hurtados no será un problema.

Mientras el GRA "evalúa iniciar obras en Escalerilla", "Cerro Verde no dará dinero para las PTAR", los agricultores de la zona de Pozo Negro, ubicado aguas arriba de Los Hurtado, "ante la negativa de los pobladores ofrecieron sus terrenos", unos días después el Congresista de la República Juan Carlos Eguren dijo que "dejaron sin presupuesto a las PTAR, por qué no se culminaron los estudios", un ciudadano diría que es "un pretexto para justificar su indolencia y obliga a otro baguazo."

El 12 de septiembre, SEDAPAR confirmó lo que ya se sabía, solo falta otorgar la viabilidad. El financiamiento y la sensibilización son los nuevos temas de agenda. Se crean comisiones, se anuncian responsables, algunos posan con cheques de más de un metro de largo para anunciar que cumplieron, en la otra orilla, Antonio Gamero, AD de Alto Selva Alegre y presidente de la AAA declara que "si no se inicia en breve la construcción de la obra rodarán cabezas".

El 31 de septiembre el AD de la Joya tomó las instalaciones de SEDAPAR, "luego de la presión ejercida por las autoridades y 4000 pobladores de La Joya... la empresa realizó las gestiones para otorgar la viabilidad", cuatro días después la respuesta de Uchumayo a la primera jornada de sensibilización no se

10 Page Vol 1, No2/2013 ABCResearch Alert 
hizo esperar, "infierno en la variante de Uchumayo, pobladores bloquearon vías", los gases lacrimógenos que irresponsablemente se lanzaron cerca a un colegio de niños, ingresa con honores a la historia del desarrollo, declaraciones a favor y en contra, pronunciamientos, editoriales, marchas, protestas, bloqueos de carreteras, contaminación, Pampa Estrella, viajes a Lima, financiamiento, el inicio de Escalerilla. "Como cangrejos" comentó un diario. SEDAPAR pediría después la "intervención de la la Iglesia y Defensoría del Pueblo", la instalación de una mesa de diálogo, en opinión de Manuel Amat, será posible "sólo si ambas partes lo piden".

El modelo de ciudad saludable que el mundo reclama y que Arequipa necesita ha sido una constante en las luchas; el AD de José Luis Bustamante y Ribero anuncia el 9 de noviembre que tiene una solución que el PD de SEDAPAR "está dispuesto a escuchar", dos días después acompañado de los AD de Yura, Sachaca, Sabandía, Socabaya proponen "que la empresa que tendrá a su cargo la obra decidirá si se construye en Hurtados o Estrella", la obra no puede parar. El Presidente del GRA interesado en la PTAR Escalerilla anuncia que "la propuesta de los seis alcaldes no se contrapone con la propuesta de

Escalerilla, la SMCV ya dio 10 millones de soles", el AD de Cerro Colorado el mismo día declara que su municipalidad "depositará 20 millones de soles en el Banco de la Nación". Arequipa no puede parar. El $A D$ de La Joya anuncia huelga, la policía envía 200 efectivos. Arequipa no puede parar. EI AD de Uchumayo hace mutis.

La oposición en las calles y la prensa se traslado al MEF, "SEDAPAR cantó victoria antes de tiempo", Roger Díaz, Director General de Programación Multianual del MEF, "retiro la viabilidad por que SEDAPAR utilizó mecanismos legales que no corresponden", "SEDAPAR pide unidad de los alcaldes para impugnar medida arbitraria del MEF", la violencia recorre todas las tiendas, "haremos respetar la viabilidad, no tengo ningún problema en dejar el cargo" dijo el PD de SEDAPAR, mientras tanto el Presidente del GRA incrementa su ofrecimiento " 45 millones de soles para Escalerilla".

En medio de denuncias y renuncias SEDAPAR convoca a los alcaldes a una reunión en un tradicional restaurante, gasto que formará parte de la historia del desarrollo, "el 75\% de los alcaldes cerró filas" todos reiteran su apoyo a la decisión técnica, en dicha reunión el alcalde opositor dijo lo de siempre, Hurtados no va y abandonó el encuentro, días después desconoció el posible acuerdo, "Ia reunión no fue oficial" dijo. Los temas de agenda cambian rápido, para el AD de Alto Selva Alegre ya no alcanza el presupuesto, "los municipios debemos aportar menos, el Canon se ha reducido".

En varios momentos de esta corta historia, diversos actores han expresado verdades, medias verdades y muchas falsedades, el 10 septiembre el especialista del CIP José Benavides declara que "la PTAR emitirá

100,000 m3 de gases ... de efecto invernadero", el 26 de octubre se publicó "Ia denuncia presentada en la II Fiscalía Penal corporativa" hecha por Dante Martínez presidente de ADUSELA, los denunciados el PD y GG de SEDAPAR, los delitos malversación de fondos y asociación ilícita para delinquir, por contratar a H\&C. El 18 de noviembre la opinión pública recordó la denuncia, "pagan 2.5 millones por un estudio que no se usa", cinco días después el autodenominado presidente de una organización con mínima presencia en la ciudad, explica a página completa, las "10 verdades sobre la PTAR", en realidad fueron algunas verdades que se pierden en un mar de mentiras, su pregonada denuncia fue archivada algunos meses después, Arequipa no puede parar, ya vendrá otra denuncia. 
En algunos momentos la historia se ha rodeado de noticias que pretendían tal vez humanizar 0 ridiculizar a los actores, como aquella del 21 de octubre de los milagros que necesita SEDAPAR y del "pedido al tatito", el ingreso del GG de SEDAPAR "a una reunión con una corbata roja brillante", o "Ios segundos nombres de los alcaldes: Florentino, Eleuterio, Mónico, Laureano" que llevaron una propuesta a la empresa. Titulares "como cangrejos", "aguas estancadas", "infierno en variante", o "los huevos de los alcaldes" del 18 de noviembre han hecho amena la historia que todos los días encontraba formas de estar presente en los medios.

El 12 de diciembre sentenciaron al AD de Uchumayo a dos años de prisión suspendida por un asunto ajeno a las PTAR, en sus interioridades culpó a SEDAPAR, al que no responsabilizó de su deseo de postular a la relección que anunció el 5 de noviembre, como efectivamente sucedió sin resultados favorables, como tampoco lo fue para el Presidente Comité de Gestión Ambiental de Uchumayo.

Ahora la lucha por Escalerilla se traslada a Lima, a fines de noviembre y por varios días se anunció el viaje de 5, 55, 80, 100 dirigentes y el AD para reunirse con el Premier, el Ministro de Economía y Finanzas, el Presidente del Congreso y cuanta autoridad tenga oficina en la capital, lo cierto es que

"Uchumayo consiguió que se baje el dedo a la PTAR". El GG de SEDAPAR declaró que el AD de Uchumayo envío un documento al MEF que influyó en la decisión, "Ia construcción de la PTAR no es prioritaria ni de emergencia", declaración que fue desmentida, aclarada y olvidada luego de varios días de estar en el llamado cotarro político.

La historia avanza y sigue siendo la misma, se pide dialogo, financiamiento, viabilidad, ejecución de Escalerilla, protestas a favor y en contra, se defiende y ataca el proyecto, se dice una cosa y se hace otra, es hora de empezar a ejecutar el proyecto. Edy Medina Presidente de la JGA de SEDAPAR se quejó de la inasistencia al fórum convocado por la empresa para explicar las ventajas y desventajas de la tecnología de tratamiento de aguas residuales, "hemos cursado más de 150 invitaciones y las ausencias solo demuestran la falta de interés en el tema", se acerca la navidad y el Fiscal de Medio Ambiente, Neil Tejada dice, luego de una reunión convocada por el Ministerio Público "alcalde de Uchumayo podría ser denunciado por oposición a los servicios públicos y delito ambiental", en esta reunión el representante del CIP "luego de escuchar los argumentos de SEDAPAR dijo que respetarán la decisión", un avance hacia el desarrollo, también lo será las tres nuevas zonas donde se podría construir la PTAR, en palabras del Presidente del GRA "serán puestas a evaluación de SEDAPAR".

Esta es una historia sin fin, circular, ciega, sorda, interesada, con mínimas posibilidades de encontrar una solución que satisfaga a todas las partes, sin un árbitro visible revestido de legalidad y legitimidad, con una ausencia interesada del gobierno central ni el MVCS ni la SUNASS, lejano a cualquiera de los intereses en discusión, los arequipeños saben quienes estuvieron ausentes en esta lucha. Tres grupos con una sub cultura claramente identificada:

1) El primero con el respaldo del conocimiento y la legalidad, que consideran y fundamentan una verdad que no puede ser cambiada sino se recurre a los mismos argumentos, este grupo sabe que es lo que necesita la población y están en capacidad de decirles cual es la solución correcta, 
tras este grupo que participa de manera distante o por terceras personas, se juntarán interés políticos que servirán para deslegitimar aquello que se pretender comunicar.

2) El segundo grupo defiende la naturaleza, el paisaje, el ornato y la no agresión por una clase que no los toma en cuenta, despierta empatía o hace aflorar odios e intereses tras una causa que en cantidad de simpatizantes la tenían perdida, pero su capacidad de movilización y presencia sistemática en los medios les aseguró aquello por lo que, con la razón de su lado o no, lucharon. Que no se construya una PTAR en su distrito.

3) El tercer grupo tiene claramente un líder, un seguidor y un grupo de activistas que han visto en el proyecto PTAR una posibilidad de consolidar su relación con un sector importante de la ciudad y de conducir una inversión importante, para ello han articulado un mensaje que sin llegar a niveles de contradicción y sin distanciarse de las otras dos posiciones distantes, poco a poco lograron fijar en la mente de las personas la necesidad de resolver primero sus problemas.

Los medios encontraron la oportunidad de construir una historia compartida, ninguno de ellos la capturó para sí, no tuvo la exclusividad, como ha sucedido en otros casos, todos privilegiaron la cobertura de noticias sin importar el mensaje o el mensajero, la única condición que sustentaba su presencia en esta historia, es que ofrecía la posibilidad de respuesta, esa es la principal característica que se destaca en todas las intervenciones, el negocio de la esperanza es el que ha marcado el camino.

Es muy fácil mantener a un pueblo desinformado, una forma de desinformar es confundir, otra forma es postergar la solución, la más ruin de todas es venderle una falsa esperanza.

Me duele tanto tu ausencia, es la letra de una canción, un poema, una cita, un recuerdo, el gobierno central nunca estuvo presente, para retirar la viabilidad no necesitaba estarlo, su presencia de defensa o la SMCV sólo apareció para anunciar que no daría dinero mientras el conflicto exista, sin recordar el grado de responsabilidad que tiene en toda esta historia, el directorio de SEDAPAR tenía un representante del GRA y otro del CIP, ambas instituciones cerca o lejos de las tres subculturas identificadas, por lo visto sus acciones si es que las tuvieron, no ayudaron a juntar las posiciones técnicas con las políticas y sociales, tampoco tuvieron éxito los directores que representan a las municipalidades. El Presidente del GRA ó el AD de Cerro Colorado, el PD de SEDAPAR al igual que el AD de Uchumayo terminaron personalizando el conflicto, en torno a sus declaraciones se movieron todos los intereses, que fueron capitalizados por el $A D$ de Cerro Colorado y los dirigentes del Cono Norte, después de tres años todos continúan en los cargos para los que fueron elegidos, con excepción, como ya se dijo, del AD de Uchumayo.

La historia sin fin tuvo lugar entre el 02 de julio del 2009 y el 31 de diciembre del 2009, desde que se dijo que la "Planta de aguas residuales tendrá nueva ubicación" y "La Joya invoca diálogo" respectivamente. Luego de algunos meses en el año 2011 se licitó la obra de Pampa Escalerilla, el 22 de julio del 2012, se inició su construcción. La a PTAR Escalerilla ha tenido una historia con fin, el PD de SEDAPAR el 26 de abril del 2012 anuncia que en "15 días comenzarían las obras", el 12 de julio reitera que "el 22 de julio se colocará la primera piedra... y que dará trabajo a 130 personas", el presidente del FREDECIR dice que "se generaran 400 puestos a favor de la región", el AD de Cerro Colorado satisfecho

13|Page Vol 1, No2/2013 ABCResearch Alert 
dijo "por fin iniciamos la construcción de la PTAR, el financiamiento está asegurado y en 1 año estará operando", el financiamiento está asegurado con los aportes del GRA, con fondos de la SMCV, las MDs de Cerro Colorado y Yura y Sedapar con un fideicomiso que compromete más del al $25 \%$ de su facturación bruta anual. Arequipa no puede parar.

La otra PTAR, la de los Hurtados ya quedó en el olvido, menudo problema después de tantas inversiones de dinero y de tiempo, ahora la SMCV ha propuesto construir una PTAR en sus terrenos, en el sitio denominado Enlozada. El actual AP de Arequipa Alfredo Zegarra declara el 06 de julio del año 2012, que "construcción de PTAR se licitará en agosto", luego de que el 2011 SEDAPAR y la SMCV firmará un convenio que justifica las futuras inversiones que destinará la minera "aportando entre 100 a 150 millones de dólares, a cambio de 1 metro cúbico por segundo de aguas tratadas, casi el 80\%. El recurso hídrico será utilizado para un proyecto de ampliación de producción mineral de la empresa". El 27 de julio el PD de SEDAPAR "aseguró que en enero del 2013 la construcción de la PTAR Enlozada debe iniciarse.", unos días después declaran que la propuesta técnica tiene observaciones

Un editorial reciente, destaca la importancia del diálogo entre las empresas privada y pública, modelo que debe ser recogido en el país, y evitar los continuos enfrentamientos.

Lejos de Arequipa se escribe una parte de esta nueva historia, el Dictamen de la Comisión de Vivienda del Congreso de la República, de la Ley de modernización de los servicios de saneamiento, facilitaría el acuerdo firmado entre las autoridades y la SMCV, en el inciso 2 del articulo 15 dice: "Los prestadores de servicios de saneamiento quedan facultados a comercializar los residuos sólidos generados en el proceso de tratamiento de agua para consumo humano y plantas de tratamiento de aguas residuales, así como el aqua residual tratada $^{10}$, con fines de reuso, cumpliendo con las normas vigentes de acuerdo a los procedimientos estableciéndose en el Reglamento de la presente Ley.

EL Congreso de la República al revisar la ley cambio una parte sustancial para esta historia, el mismo artículo, el mismo inciso dice: "Los prestadores de servicios de saneamiento quedan facultados a comercializar los residuos sólidos generados en el proceso de tratamiento de agua para consumo humano y plantas de tratamiento de aguas residuales tratadas con fines de reuso, cumpliendo con las normas vigentes de acuerdo a los procedimientos estableciéndose en el Reglamento de la presente Ley".

El ejecutivo ha devuelto el proyecto de Ley. La historia continúa.

10 El subrayado es nuestro

14 Page Vol1, No2/2013 ABCReserch Alert 
Anexo I: Acrónimos y personalidades

AAA Asociación de Alcaldes de Arequipa

AD Alcalde Distrital

ADUSELA Asociación de Usuarios del Servicio de Electricidad

AP

Alcalde Provincial

AUPA

Asociación de Urbanizaciones Populares de Arequipa

CC

Cámara de Comercio

CCA

Colegio de Arquitectos de Arequipa

CIP

Colegio de Ingenieros del Perú

COFREN Central de Organizaciones y Frentes de Arequipa

DGPP

CGA

Dirección General de Programación Multianual del MEF

FACA

Comité de Gestión Ambiental

FDTA

Frente Amplio Cívico de Arequipa

FREDICON

Federación de Trabajadores de Arequipa

FREDECIR

Frente de Desarrollo e Integración del Cono Norte

GG

Frente de Desarrollo del Área Central Cono Norte

GRA

Gerente General

$H \& C$

Gobierno Regional de Arequipa

JGA

Hugo Campusano Consultores

$M D$

Junta General de Accionistas

$M P$

Municipalidad Distrital

MVCS

Municipalidad Provincial

PT

Ministro de Vivienda Construcción y Saneamiento

PTAR

Presidente del Directorio

PTAP

Planta de Tratamiento de Aguas Residuales

SMCV

Planta de Tratamiento de Agua Potable

SEDAPAR

Sociedad Minera Cerro Verde

SUNASS

Empresa Prestadora de Servicios de Agua y Saneamiento de Arequipa

Superintendencia Nacional de Servicios de Saneamiento 


\section{Personajes}

\begin{tabular}{|c|c|}
\hline Alfredo Zegarra & AD de José Luis Bustamante y Rivero \\
\hline Antonio Gamero & AD de Alto Selva Alegre \\
\hline Berly Gonzales & Gerente General del GRA \\
\hline Ciro Rojas & Decano del CAA \\
\hline Clark Velando & vocero del CIP \\
\hline Dante Martínez & Presidente de ADUCELA \\
\hline David Inofuente & AD de La Joya \\
\hline Edmundo Laguna & FREDICON \\
\hline James Fernández & Presidente del Directorio de SEDAPAR \\
\hline Jorge del Castillo & Primer Ministro \\
\hline José Benavides & Especialista del CIP \\
\hline José Pinto & Decano del CIP \\
\hline Juan Manuel Guillen & Presidente del GRA \\
\hline Juan Valdivia Romero & Ministro de Energía y Minas \\
\hline Gerónimo López & FDTA \\
\hline Guillermo León & Vice Ministro de Saneamiento del MVCS \\
\hline Hernán Alegre & AD de Uchumayo \\
\hline Hugo Rosand & Director de SEDAPAR \\
\hline Hugo Campuzano & Gerente General de H\&C Consultores \\
\hline Humberto Olaechea & CCGA de Uchumayo \\
\hline Leandro Castañeda & AUPA \\
\hline Luis Aguirre & AD de Miraflores \\
\hline Oscar Achata & Gobernador Regional \\
\hline Oscar Pastor & Gerente General de SEDAPAR \\
\hline Manuel Amat & Defensor del Pueblo, Arequipa \\
\hline Manuel Vera & AD de Cerro Colorado \\
\hline Mario Jacobo, & presidente del FREDECIR \\
\hline Máximo Mendoza & COFREN \\
\hline Miguel Ocharán & Jefe de Planificación del GRA \\
\hline Ramón Pachas & FACA \\
\hline Roger Díaz & Director General de Programación Multianual del MEF \\
\hline Simón Balbuena & AD de Hunter y Presidente de la AAA, posteriormente AP de Arequipa \\
\hline Yamel Romero & AP de Arequipa \\
\hline
\end{tabular}




\section{Anexo II: Los olvidos se olvidan cuando sus recuerdos se hacen necesarios}

En el tiempo se suscribieron los siguientes acuerdos:

1) El Acuerdo de la Mesa de Diálogo entre el Comité de Lucha por la Defensa de los Intereses de Arequipa se firmo el 02 de agosto del 2006, allí se acordó que la SMCV elabore los Expedientes Técnicos de las PTAP y PTAR, conocidos los presupuestos de ambas obras, el financiamiento será asumido proporcionalmente por la SMCV y las municipalidades. Intervinieron Jorge del Castillo y Juan Valdivia Romero, Premier y Ministro de Energía y Minas, Yamel Romero y Simón Balbuena como AP de Arequipa y Presidente de la AAA y los representantes de AUPA, FDTA, FACA, COFREN, FREDICON y el CCGA de Uchumayo: Leandro Castañeda, Gerónimo López, Ramón Pachas, Máximo Mendoza, Edmundo Laguna y Humberto Olaechea.

2) El Acuerdo entre el Gobierno Regional de Arequipa, Municipalidad Provincial de Arequipa, Municipalidades Distritales, Comité de Lucha de Arequipa y la Sociedad minera Cerro Verde, suscrito el 16 de mayo del 2007, con la intervención de Jorge del Castillo y Lourdes Mendoza, Premier y Vicepresidente de la República, Juan Manuel Guillén, Simón Balbuena y Justo Mayta, Presidente Regional, Alcalde Provincial y Presidente de la Asociación de Alcaldes, diversos alcaldes distritales y representantes del Comité de Lucha y Jorge Benavente por la SMCV, se acordó que se coordinará con SEDAPAR la elaboración los Expedientes de las dos Plantas a nivel de Perfil, el GR se compromete su participación en el financiamiento con intervención del Ministerio de Economía y Finanzas y el Banco de la Nación, acuerdan que Cerro Verde asuma la construcción de la PTAP, con cargo a los recursos de Canon Minero futuro, lo cual a su vez se deducirá a las Municipalidades de Arequipa.

3) El Acta de Acuerdo para el financiamiento de las Plantas de Tratamiento de Aguas Servidas, se firmó en Arequipa el 22 de julio de 2008, participaron el Presidente Regional, Alcaldes Provincial y Distritales y SEDAPAR, acordaron estructurar el esquema de Co-financiamiento no reembolsable que se ejecutará en los periodos presupuestales entre los años 2008-2011 como sigue:

$\begin{array}{rlr}\text { i. } & \text { SEDAPAR } & \text { S/. 80.0 MM } \\ \text { ii. } & \text { MVCS } & \text { S/.73.4 MM } \\ \text { iii. } & \text { Gobierno Regional de Arequipa: } & \text { S/. 75.0 MM } \\ \text { iv. Gobiernos Distritales de Arequipa: } & \text { S/. } 60.0 \mathrm{MM} \\ \text { v. Gobierno Provincial de Arequipa: } & \text { S/. } 11.6 \mathrm{MM}\end{array}$

4) El 06 de julio del 2009 el Directorio de Sedapar acuerda "la operación y constitución de un Fideicomiso para la ejecución de Proyecto, así como, autorizar la transferencia en dominio Fiduciario de los recursos dinerarios provenientes de una operación de endeudamiento de $\mathrm{S} /$. 80 MM". Sucesivos documentos darán cuenta después de la imposibilidad de la empresa de asumir con recursos propios su participación en el financiamiento de la Planta, así requerirá al Ministerio de Vivienda Construcción y Saneamiento amplíe el monto que nunca se comprometió formalmente, a la Superintendencia de Servicios de Saneamiento para que autorice el uso de fondos propios, a la SMCV para que aporte la diferencia entre ambas obras, al Banco de la Nación y otras entidades internacionales para que evalúen diversos modelos de financiamiento. 